\section{Cureus}

Received 05/25/2019

Review began 07/12/2019

Review ended 07/28/2019

Published 08/10/2019

\section{(C) Copyright 2019}

Yazigi et al. This is an open access article distributed under the terms of the Creative Commons Attribution License CC-BY 3.0., which permits unrestricted use, distribution, and reproduction in any medium, provided the original author and source are credited.

\title{
Granulomatous Mastitis: A Rare Case with Sjogren's Syndrome and Complications
}

\author{
George Yazigi ${ }^{1}$, Becca H. Trieu ${ }^{2}$, Michael Landis ${ }^{2}$, Jignesh G. Parikh ${ }^{3}$, Mamta Mangal ${ }^{4}$ \\ 1. Internal Medicine, University of Central Florida College of Medicine, Orlando, USA 2. Miscellaneous, \\ University of Central Florida College of Medicine, Orlando, USA 3. Pathology, Orlando VA Medical \\ Center, Orlando, USA 4. Internal Medicine, University of Central Florida College of Medicine/Orlando VA \\ Medical Center, Orlando, USA
}

$\square$ Corresponding author: George Yazigi,dr.g.yazigi@gmail.com

Disclosures can be found in Additional Information at the end of the article

\section{Abstract}

Granulomatous mastitis (GM) is a rare benign chronic inflammatory process of the breast in reproductive aged females. Although considered idiopathic in many cases, it has been associated with other conditions. Herein we report a highly complex and interesting case of GM in a young female with Sjogren's syndrome. We also review the literature and discuss challenges pertaining to the management of patients with similar risk factors. According to our knowledge, this is the third case documenting the co-occurrence of GM and Sjogren's syndrome. We focus on the challenges and complications of GM in the context of an autoimmune disease. With evidence from our patient's disease course and support from the literature, we advocate the use of corticosteroids for GM to prevent complications in patients with additional risks factors such as an autoimmune disease.

Categories: Family/General Practice, Internal Medicine, Rheumatology

Keywords: granulomatous mastitis, sjogren's syndrome, mastitis

\section{Introduction}

Idiopathic granulomatous mastitis (IGM) is a rare benign chronic inflammatory process of the breast with an unknown and debatable etiology [1]. First discussed in the literature in 1972 by Kessler and Wolloch, IGM was originally described as having a pathogenesis revolved around an autoimmune process [2]. IGM typically presents as a unilateral painful breast mass in a reproductive aged female, with a median age of diagnosis in the mid-30s [1,3-4]. Other possible presenting findings may include nipple discharge, nipple retraction, fistula formation, and skin hyperemia [1,5]. If there is a suspected cause of disease, IGM would be called granulomatous mastitis (GM). Both IGM and GM present similarly to other more concerning conditions such as carcinoma and infection $[1,5]$. Clinical findings and imaging for GM are nonspecific and overlap with other diagnoses, which could delay the treatment [5]. A definitive diagnosis of IGM requires a biopsy with histopathologic evidence of inflammatory infiltrates and epithelioid histiocytes defining noncaseating granulomas [6]. Even with a biopsy of noncaseating granulomatous inflammation, a group of potential causes must be excluded to make a diagnosis of IGM versus GM. Some of these may include well-known causes of granulomas like sarcoidosis and Mycobacterium tuberculosis infection [7]. This becomes significant as some believe the high amount of reports of IGM diagnoses from developing countries could be a misdiagnosis of GM due to tuberculosis.

Given the low prevalence of GM, there has not been a development for a consistent treatment 


\section{Cureus}

guideline [3]. Treatments that have shown efficacy include observation, antibiotics, excision, and corticosteroids [3-4]. The difficulty in treating GM could potentially lead to complications such as fistula formation, abscesses, post-procedural infections, and recurrences [7]. In our case, we will be discussing the complications subsequent to a diagnosis of GM in a patient with existing Sjogren's syndrome which was hypothesized to be associated with GM but is underreported in the literature.

\section{Case Presentation}

The patient is a 37-year-old Hispanic female who initially presented for a firm mass on her right breast that had enlarged and became painful over one year. Her medical history is significant for Sjogren's syndrome with no systemic treatment, hypothyroidism, parity, a right breast benign adenoma, and bilateral breast augmentation with subsequent removal of the implants due to infection. A fine-needle aspiration (FNA) biopsy showed GM (Figure 1). Right axillary lymph node contained lymphoid tissue with sinus histiocytosis. There was no evidence of cancer, fungus, or tuberculosis. A diagnosis of idiopathic GM was made. However, no treatment was given as she was lost to follow-up.

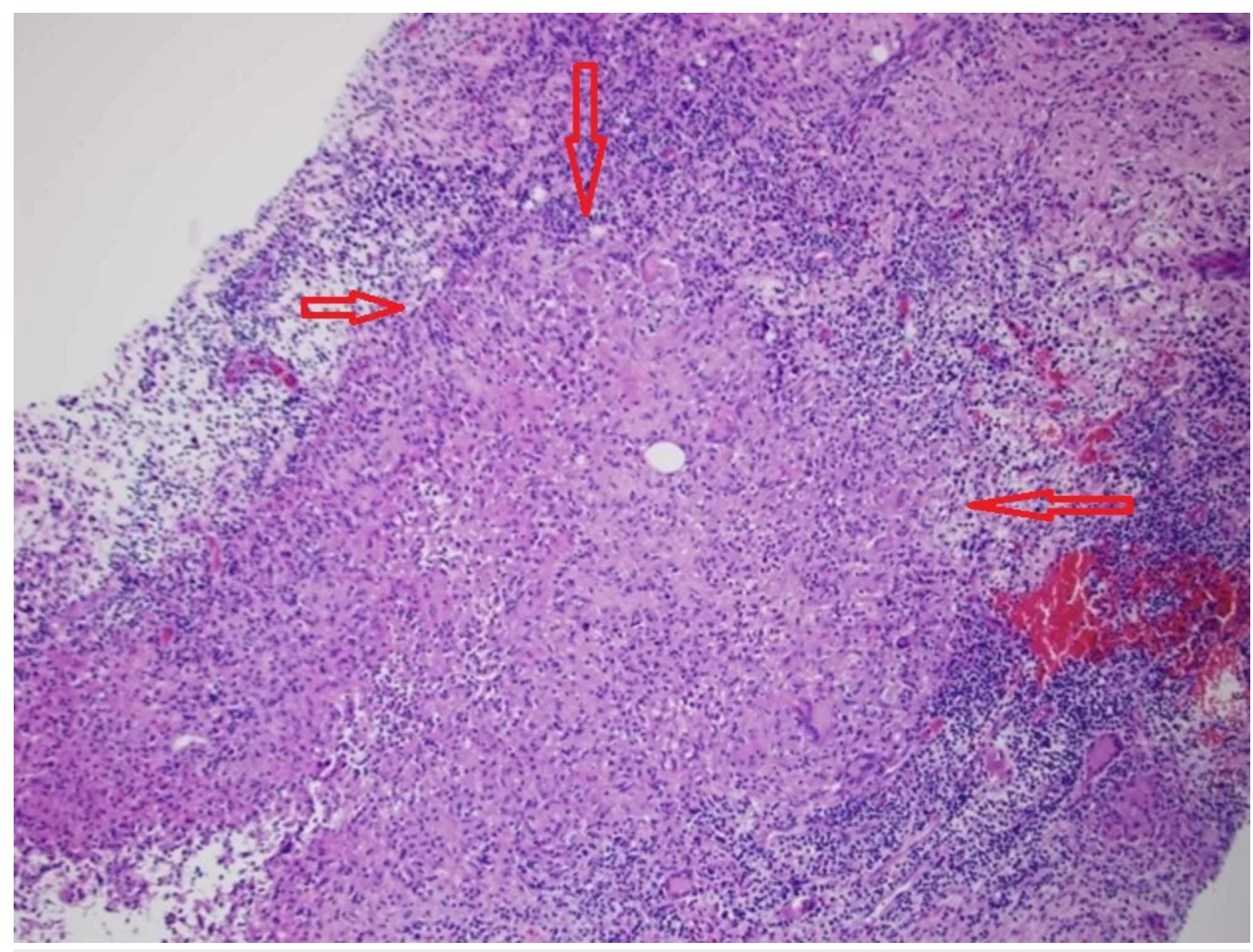

\section{FIGURE 1: Breast biopsy}

Breast biopsy shows noncaseating granulomatous inflammation, consistent with granulomatous mastitis (between red arrows).

Three months after the FNA, her incision site did not fully heal while the mass continued to grow. There was worsening erythema, induration, and pain associated with the mass. Ultrasound (US) of the mass showed complex partially fluid-filled parenchymal changes directly under the indurated area with extension into the biopsied GM lesion suspicious for fistularization with possible abscess or phlegmon formation (Figure 2). US findings did not indicate that the mass was drainable. Although her labs did not indicate an active infection, the 


\section{Cureus}

patient was placed on oral linezolid $400 \mathrm{mg}$ twice daily for 14 days and asked to follow-up in the outpatient surgical clinic.

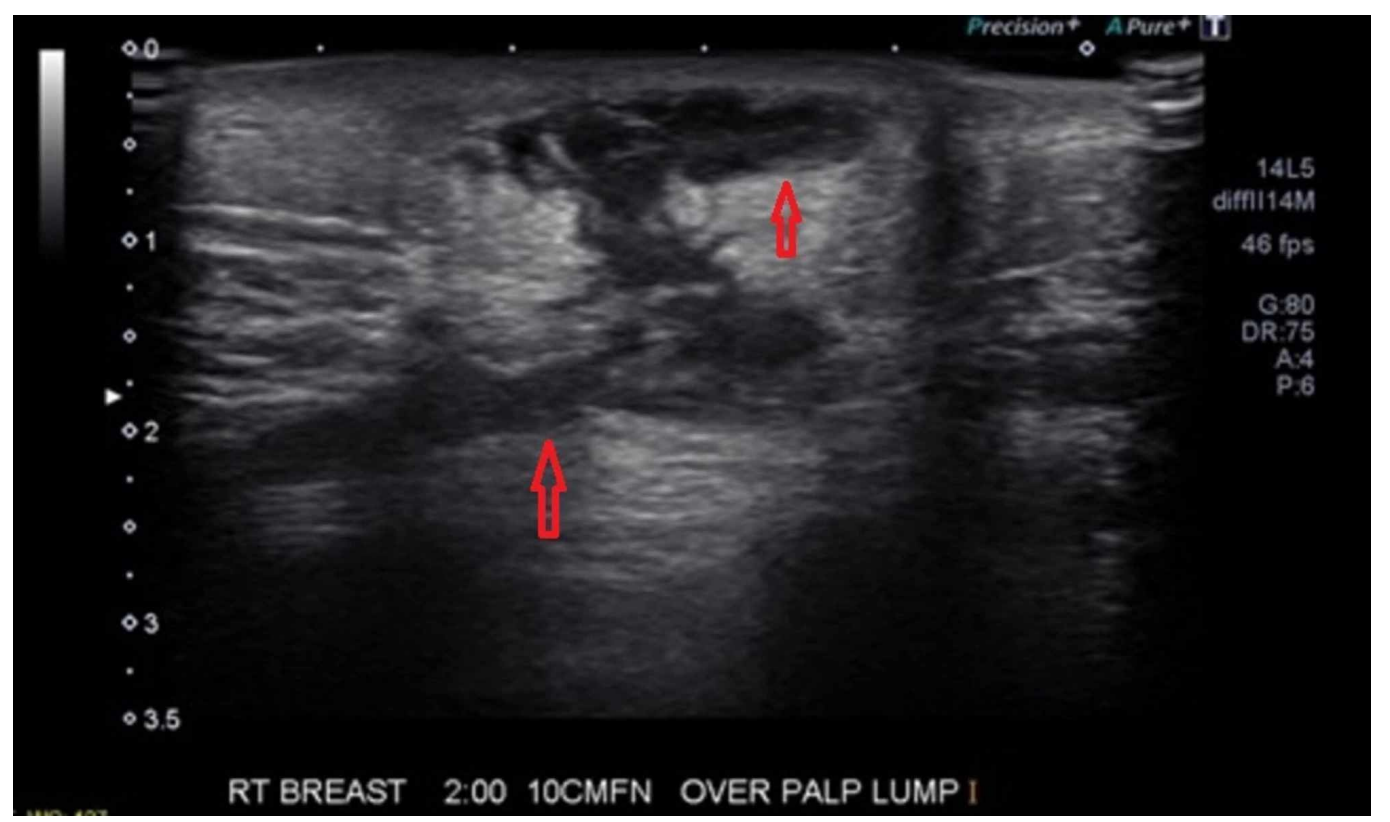

\section{FIGURE 2: Breast ultrasound}

Breast ultrasound (US) before incision and drainage (I\&D) shows complex partially fluid-filled parenchymal changes directly under the indurated area (upper red arrow) with extension into the biopsied granulomatous mastitis lesion suspicious for fistularization with possible abscess or phlegmon formation (lower red arrow).

Prior to the completion of her antibiotic course, she presented with thick drainage from the site of the mass. This presentation was consistent with a breast abscess, for which the patient underwent an incision and drainage (I\&D) with packing. During the post-op evaluation, the site of I\&D showed granulation and sanguineous drainage without any signs of infection which was supported by her labs and vital signs. Post-I\&D US showed resolution of parenchymal fluid and confirmation of a persistent path between the I\&D site and the biopsied GM lesion (Figure 3). Differential diagnoses from US included progression of GM with fistularization and a superimposed infection with more evidence for the former diagnosis. One notable drawback during the investigation for her case is the lack of a bacterial culture; hence, we could not definitively rule out infection as a complication associated with her GM. A week after I\&D, the patient reported some improvement in the I\&D site. 


\section{Cureus}

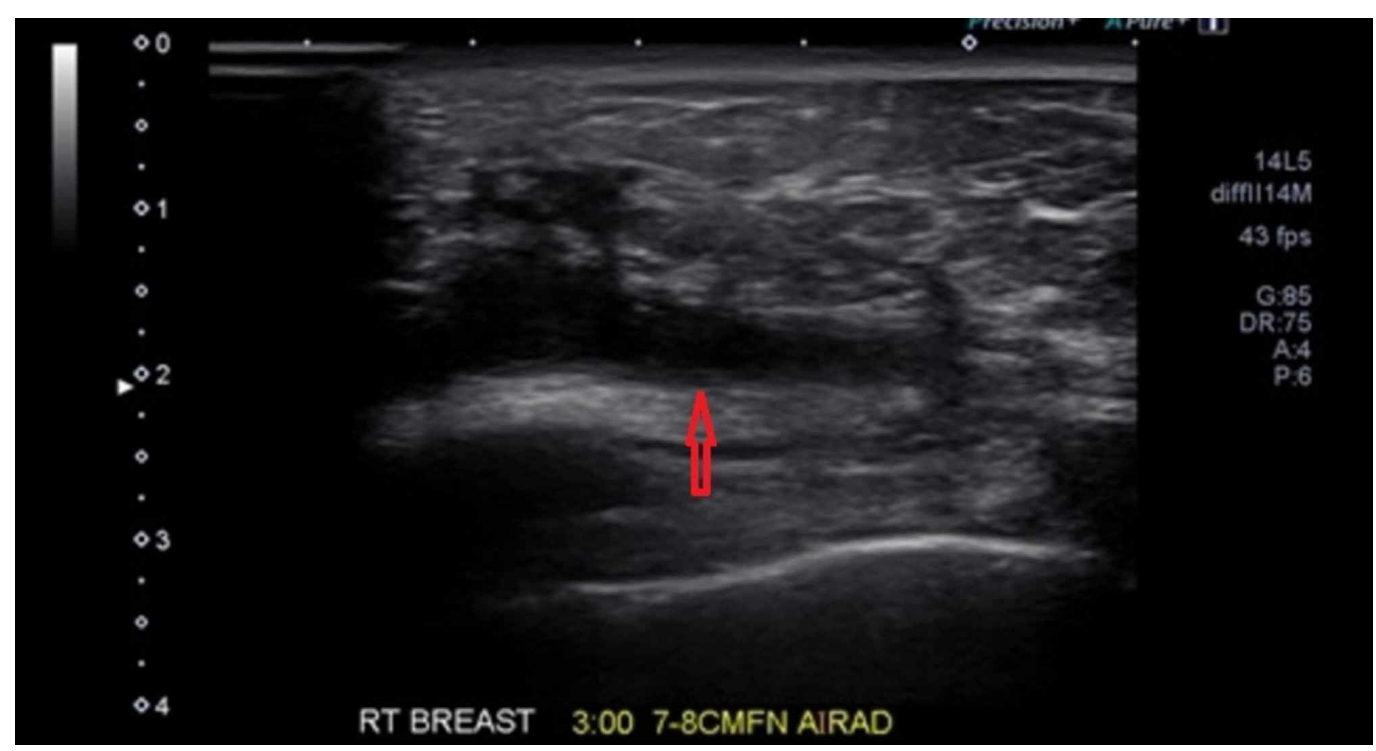

\section{FIGURE 3: Breast ultrasound}

Breast ultrasound (US) post incision and drainage (I\&D) shows resolution of parenchymal fluid and confirmation of a persistent path between the I\&D site and the biopsied granulomatous mastitis lesion (red arrow).

\section{Discussion}

IGM is a poorly understood condition with only a few hundred cases reported worldwide [8]. IGM has been suggested to have a $1.8 \%$ incidence among females presenting with breast pathology [5]. Many different risk factors have been proposed for the development of IGM. Prior pregnancy, autoimmune conditions, infection, hyperprolactinemia, and oral contraceptives have all been proposed as potential contributing factors [9-10]. There is a higher incidence in Hispanic women, as was our patient [4]. Autoimmunity has been viewed as a main cause of GM due to the favorable response of the condition to corticosteroids [2]. Additionally, certain other risk factors such as pregnancy, smoking, abscess formation, and lactation have been discussed as increasing the risk for recurrence [3,11]. One study has shown up to a $50 \%$ recurrence rate [12]. However, amongst these factors, the etiology of autoimmune versus infectious is still debatable due to a lack of consistent findings [7,13-14].

Our patient presented with risk factors that historically have been considered related to the development of GM. These factors include her age, race, history of pregnancy, Sjogren's syndrome, and a potentially autoimmune hypothyroidism. Sjogren's syndrome is an autoimmune disease that affects exocrine glands, mainly leading to infiltration of the lacrimal and salivary glands. Although it has been hypothesized that GM is associated with other autoimmune conditions, one of which is autoimmune thyroiditis, there is little solid evidence for its association with Sjogren's syndrome [2,15-16]. As Sjogren's affects exocrine glands, it is also possible for mammary glands to be affected. Our case further reinforces the rare finding of a possible association with Sjogren's. Her Sjogren's was diagnosed by an ophthalmologist who managed it solely with dry-eye treatments. No treatment with steroids was given after biopsy showed GM since she was lost to follow-up.

Due to the low incidence and lack of standardized treatment guidelines for GM, many physicians are unfamiliar with treating this condition. A case series with only nine cases has shown that $50 \%$ of patients with IGM spontaneously resolved breast masses without any treatments [6]. Although close observation is a possible approach for GM, a lack of treatment 
may have contributed to our patient's complex course of disease. Literature has shown that trauma such as an FNA can increase the risk for complications [7,17]. Looking at our patient's case retrospectively, we believe steroid therapy may have prevented her abscess and fistula.

Abscess and fistula development are possible sequelae of GM [10]. Our patient was found to have a fistula with possible abscess on ultrasound following an FNA that was done three months prior. In a 2006 retrospective chart review, only one of the 21 cases of GM presented with fistula formation [14]. A study in Turkey of 63 patients found $45.5 \%$ with abscess formation [18]. GM has no optimal treatment, and the development of abscesses and/or fistula make the disease even more difficult to treat as wide local excision may not be successful and could be disfiguring $[4,19]$. A compounding difficulty related to fistula and abscesses is indicated by research showing that they are predictors for recurrences [18]. A study in Jordan shows successful conservative treatment of fistulae in IGM with medications instead of surgery [20]. This compilation of evidence further reinforces our preference for corticosteroids in cases like our patient's.

\section{Conclusions}

This case offers more support for the rare-documented association between Sjogren's syndrome and GM. To our knowledge, this is the third instance to show such an association. In our patient's case, the word "idiopathic" should be retracted when Sjogren's syndrome is the suspected cause of her GM. This paper advocates the use of corticosteroids in a subpopulation of patients with GM who have risk factors resembling the case presented. As an autoimmune condition likely caused and attenuated our patient's GM, she was likely placed at a higher risk of complications when enduring trauma via FNA without subsequent steroid therapy. To prevent these complications, patients with similar risk factors should be placed on corticosteroids right after their FNA result shows GM. Once developed, abscess and fistula formation complicate the management and surgical excision of GM. It conveys worsened prognosis with higher recurrence rates. The complications may respond well to corticosteroid treatments which further supports our recommendation for their use. Further research, especially randomized-control studies with a larger sample size, should be conducted to explore optimal treatments for patients with different risk profiles.

\section{Additional Information}

\section{Disclosures}

Human subjects: Consent was obtained by all participants in this study. Conflicts of interest: In compliance with the ICMJE uniform disclosure form, all authors declare the following: Payment/services info: All authors have declared that no financial support was received from any organization for the submitted work. Financial relationships: All authors have declared that they have no financial relationships at present or within the previous three years with any organizations that might have an interest in the submitted work. Other relationships: All authors have declared that there are no other relationships or activities that could appear to have influenced the submitted work.

\section{References}

1. Barreto DS, Sedgwick EL, Nagi CS, Benveniste AP: Granulomatous mastitis: etiology, imaging, pathology, treatment, and clinical findings. Breast Cancer Res Treat. 2018, 171:527-534. 10.1007/s10549-018-4870-3

2. Kessler E, Wolloch Y: Granulomatous mastitis: a lesion clinically simulating carcinoma . Am J Clin Pathol. 1972, 58:642-646. 10.1093/ajcp/58.6.642

3. Mohammed S, Statz A, Salmans Lacross JA, et al.: Granulomatous mastitis: a 10 year experience from a large inner city county hospital. J Surg Res. 2013, 179:194. 
10.1016/j.jss.2012.10.244

4. Pandey TS, Mackinnon JC, Bressler L, Millar A, Marcus EE, Ganschow PS: Idiopathic granulomatous mastitis-a prospective study of 49 women and treatment outcomes with steroid therapy. Breast J. 2014, 20:258-266. 10.1111/tbj.12263

5. Baslaim MM, Khayat HA, Al-Amoudi SA: Idiopathic granulomatous mastitis: a heterogeneous disease with variable clinical presentation. World J Surg. 2007, 31:1677-1681.

10.1007/s00268-007-9116-1

6. Lai ECH, Wing Cheong C, Ma TKF, Tang APY, Poon CSP, Heng Tat L: The role of conservative treatment in idiopathic granulomatous mastitis. Breast J. 2005, 11:454-456. 10.1111/j.1075122X.2005.00127.X

7. Fletcher A, Magrath IM, Riddell RH, Talbot IC: Granulomatous mastitis: a report of seven cases. J Clin Pathol. 1982, 35:941. 10.1136/jcp.35.9.941

8. Wilson JP, Massoll N, Marshall J, Foss RM, Copeland EM, Grobmyer SR: Idiopathic granulomatous mastitis: in search of a therapeutic paradigm. Am Surg. 2007, 73:798-802.

9. Akcan $\mathrm{A}, \mathrm{Oz} \mathrm{AB}$, Dogan $\mathrm{S}$, et al.: Idiopathic granulomatous mastitis: comparison of wide local excision with or without corticosteroid therapy. Breast Care (Basel). 2014, 111:115. $10.1159 / 000360926$

10. Zhou F, Yu LX, Ma ZB, Yu ZG: Granulomatous lobular mastitis. Chronic Dis Transl Med. 2016, 2:17-21. 10.1016/j.cdtm.2016.02.004

11. Uysal E, Soran A, Sezgin E: Factors related to recurrence of idiopathic granulomatous mastitis: what do we learn from a multicentre study?. ANZ J Surg. 2018, 635.

10.1111/ans.14115

12. Joseph K-A, Luu X, Mor A: Granulomatous mastitis: a New York public hospital experience . Ann Surg Oncol. 2014, 21:4159-4163. 10.1245/s10434-014-3895-Z

13. Tse GMK, Poon R, Ramachandram K, et al.: Granulomatous mastitis: a clinicopathological review of 26 cases. Pathology. 2004, 36:254-257. 10.1080/00313020410001692602

14. Akcan A, Akyildiz H, Deneme MA, Akgun H, Aritas Y: Granulomatous lobular mastitis: a complex diagnostic and therapeutic problem. World J Surg. 2006, 30:1403-1409.

10.1007/s00268-005-0476-0

15. Letourneux C, Diemunsch P, Korganow AS, Akladios CY, Bellocq JP, Mathelin C: First report of granulomatous mastitis associated with Sjögren's syndrome. World J Surg Oncol. 2013, 268. 10.1186/1477-7819-11-268

16. Vinayagam R, Cox J, Webb L: Granulomatous mastitis: a spectrum of disease . Breast Care (Basel). 2009, 4:251-254. 10.1159/000229541

17. Pluguez-Turull CW, Nanyes JE, Quintero CJ, Alizai H, Mais DD, Kist KA, Dornbluth NC: Idiopathic granulomatous mastitis: manifestations at multimodality imaging and pitfalls . Radiographics. 2018, 38: 330-356. 10.1148/rg.2018170095

18. Yilmaz T, Gurel B, Ata Guler S, Ali Baran M, Ersan B, Duman S, Utkan Z: Scoring idiopathic granulomatous mastitis: an effective system for predicting recurrence?. Eur J Breast Health. 2018, 14:112-116. 10.5152/ejbh.2018.3709

19. Kiyak G, Dumlu EG, Kilinc I, et al.: Management of idiopathic granulomatous mastitis: dilemmas in diagnosis and treatment. BMC Surg. 2014, 14:66-66. 10.1186/1471-2482-14-66

20. Almasad JK: Mammary duct fistulae: classification and management. ANZ J Surg. 2006, 76:149-152. 10.1111/j.1445-2197.2006.03611.x 